\title{
REVIEW
}

\section{Optical coherence tomography and its use in optical neuritis and multiple sclerosis}

\author{
Kemenyova $\mathrm{P}^{1}$, Turcani $\mathrm{P}^{1}$, Sutovsky $\mathrm{S}^{1}$, Waczulikova $\mathrm{I}^{2}$ \\ 1st Department of Neurology, Faculty of Medicine, Comenius University and University Hospital, \\ Bratislava, Slovakia.pkemenyova@gmail.com
}

\begin{abstract}
Optical coherence tomography is a relatively new non-invasive imaging technique used for obtaining the images and quantifying the layers of the retina. It also provides information about optic nerve head topography, peripapillary retinal nerve fiber layer thickness, and macular volume which correlates with axonal loss. Until now, this method was used mainly in ophthalmology; now it has emerged as relevant in neurology as well. RNFL thickness is of particular interest in optic neuropathies and in multiple sclerosis. In sclerosis multiplex, axonal loss occurs as early as the first stages and the quantification of the RNFL thickness by OCT provides an indirect measure of axonal and neuronal loss in the anterior visual pathways. Because OCT is noninvasive, easy to obtain, and highly reproducible, it can be used as a marker of axonal loss and as an endpoint in clinical trials. This paper presents a comprehensive summary of the use of this new diagnostic method in multiple sclerosis patients (Fig. 1, Ref. 58). Text in PDF www.elis.sk.

Key words: optical coherence tomography, optical neuritis, multiple sclerosis.
\end{abstract}

\section{What is optical coherence tomography?}

Optical Coherence Tomography (OCT) is a relatively new diagnostic method. It is a non-contact method with a great therapeutic potential, as well. Owing to its expedience, safety and a relatively low purchasing and running cost, it is used more and more in basic medical research as well as in clinical practice. Until now, this method was used mainly in ophthalmology; however, in the last ten years, it has been increasingly used in some fields of neurology.

One advantage of OCT is its ability to capture the image of biological tissue with high lateral resolution, based on the detection of reflection delay and intensity of the reflected light, with lateral resolution decoupled from axial resolution. Other advantages include its ability to render tissue structures even in highly diffusive environments, in real time, and with a resolution of 1-15 $\mu \mathrm{m}$, which is one or two degrees higher than the resolution of conventional imaging methods like ultrasound, magnetic resonance and computer tomography. The device offers a large number of scan lines in different imaging modes and options to construct a $3 \mathrm{D}$ reconstruction of the retina and carry out precise metric measurements and volume calculations.

The quantification of the retinal nerve fiber layer (RNFL) thickness through OCT is an indirect measure of axonal and neu-

${ }^{1} 1$ st Department of Neurology, Faculty of Medicine, Comenius University and University Hospital, Bratislava, Slovakia, and ${ }^{2}$ Department of Nuclear Physics and Biophysics, Division of Biomedical Physics Faculty of Mathematics, Physics and Informatics, Comenius University, Bratislava, Slovakia

Address for correspondence: P. Kemenyova, MD, 1st Dept of Neurology, Faculty of Medicine, Comenius University and University Hospital, Mickiewiczova 13, SK-813 69 Bratislava 1, Slovakia. Phone: +421.2 .57290452$ ronal loss in the anterior visual pathways. Axonal degeneration in multiple sclerosis (MS) occurs as a result of inflammation and demyelination in the early stages of the disease $(1,2)$. Axonal loss is the reason behind a large part of permanent invalidity in MS patients, which is why the monitoring of axonal damage has become a priority in MS and OCT; it is a sensitive, precise and reproducible technique and is gaining greater importance and diagnostic significance in neurology as well (3).

\section{The principles of optical coherence tomography}

The basic principle of OCT imaging is based on the optical technique known as Michelson low-coherence interferometry. It uses infrared light with a short coherent length, whose source is a luminescent diode. The interferometer measures phase differences between the reflected control ray (reflection from the reference mirror) and the ray reflected from the tissue microstructure; the aim is to obtain a two- or three-dimensional tomography image. The principle of OCT is very similar to the ultrasound method, the only difference being that OCT works with light instead of acoustic waves (4). OCT compares the delay of tissue reflection with referential reflection. The principle of image creation lies in lateral scanning of the light ray, creation of axial scans (A-scans), and subsequent combination of A-scans into the resulting image (B-scan). Thus, each A-scan carries information about the strength of the reflected signal, or the depth of the scanned tissue.

OCT works with the wavelength of infrared lights, which eliminates the risk of glare. In creating the image of the retina, the wavelength of the light is within the range of 800-900 $\mathrm{nm}(5,6)$.

The in vivo OCT method was first used to measure a retina in 1993 (7) and since then, four new generations of OCT devices have 
been developed. The resolution of OCT is high, in the range of 3 to $20 \mu \mathrm{m}$. Therefore, it has the ability to create images of very small optic structures. The third generation time domain OCT devices" resolution is in the range of $10-15 \mu \mathrm{m}$; the newest, fourth generation of spectral domain OCT devices pushes the quality of image detail even further and their resolution is at the level of 3 to $7 \mu \mathrm{m}$. Spectral OCT enables, among other things, the fast scanning of a large amount of data, combining OCT with other imaging techniques into a single image, three-dimensional reconstruction, differentiation of individual layers of the retina, and better visualization $(5,6)$.

In examination using the OCT, pupillary dilation is not always necessary, as a three-millimeter pupil is adequate for obtaining the image. Good cooperation of the patient can help prevent distortion caused by eye movement. The results obtained from the exam are compared with the normative database which contains data from people considered as a representative specimen of a healthy population.

\section{The eye as a model of neurodegenerative changes in multiple sclerosis}

The most significant visualized structure in neurological diseases is the retinal nerve fiber layer (RNFL), which is the innermost layer of the retina. RNFL is composed mainly of axons of ganglion cells which are not myelinated; due to this, the retina is the only part of the CNS which can be visualized directly $(8,9$, 10). OCT, then, enables us to evaluate the impact of the pathologic process on the axons and nerve fibers. Acute optical neuritis occurs in a one of the stages of multiple sclerosis in as many as 30 to 70 $\%$ of patients $(11,12)$. Subclinical chronic optical neuropathy is also observed in some patients. The tendency of MS to affect the optic nerve is very high. In post mortem analysis, MS lesions on the optic nerve were discovered in $94-99 \%$ of specimens from MS patients, irrespective of the history of clinical acute optical neuritis $(13,14)$.

In addition to providing data about axon integrity through the measuring of RNFL thickness, OCT is also able to measure total macular volume (TMV). As the outer macula is well supplied with ganglion cell bodies, it seems that OCT evaluation of the outer macula may be able to provide an estimate of neuron integrity (mainly the ganglion cell integrity) in the retina, as well $(15,16)$.

The average thickness of the RNFL in healthy subjects up to 15 years of age is approximately $110-120 \mu \mathrm{m}$; with aging, the thickness is reduced by about $0.017 \%$ per year, which is equivalent to the reduction of retina thickness by $10-20-\mu$ m over 60 years (17).

\section{OCT and optical neuritis}

\section{Pilot studies}

Optical neuritis is one of the manifestations and the second most frequent symptom of MS. Studies focusing on the treatment of optical neuritis have shown that the risk of the development of MS in patients with a diagnosed first episode of inflammation of the optic nerve is $50 \%$ during the following 15 years. The risk rises to $72 \%$ in patients with at least one demyelinating lesion visible in the MR image (18).
The first study documenting findings from OCT eye measurements in MS patients was published in 1999. It showed that the average RNFL thickness in patients with MS and optical neuritis is much smaller than in healthy subjects. RNFL thickness was reduced by an average of $46 \%$ in the group with optical neuritis vs. the group of control subjects, and by an average of $28 \%$ in comparison with the patient's opposite, unaffected eye (19).

The authors of the study from 2005 have confirmed a $33 \%$ reduction of RNFL thickness in the affected eyes of those patients who have suffered from acute optical neuritis vs. control subjects' eyes, and an average $27 \%$ reduction in the comparison of affected and unaffected eyes of the same patient. In addition, they discovered that in patients with MS and optical neuritis, the macular volume measured after the optical neuritis episode was also significantly lower. They proved that the thinning of the RNFL correlated more with the visual evoked potentials of the $\mathrm{P} 100$ wave amplitude (axonal integrity image) than the latency of the P100 wave (myelin integrity image) (20); this supports the hypothesis that RNFL thinning reflects axonal degeneration.

\section{RNFL thickness in acute optical neuritis}

In 2006, Pro et al (21) showed that RNFL becomes significantly thinner as early as 2 to 4 months after the outburst of optical neuritis. They also proved that RNFL measured at the baseline visit was slightly thicker in the eye with acute optical neuritis as opposed to the unaffected eye even though ophthalmoscopic exams of the patients did not uncover any cases of evident edema of the optic disc. These studies suggest that OCT is able to acutely diagnose even very slight disc edemas in optical neuritis. Therefore, it is good to be aware of the time elapsed between the loss of eyesight and the interpretation of OCT measurement (22).

\section{The development of RNFL thickness after recovery from isolated optical neuritis}

Most often, the thinning of RNFL was observed through OCT in the 3rd - 6th month after the development of optical neuritis $(23,24)$. In about $75 \%$ of MS patients, the reduction of RNFL thickness in the affected eye by $10-40 \mu \mathrm{m}$ takes place during the first 3-6 months after the end of acute optical neuritis. This suggests that significant and fast axon degeneration follows immediately after the primary demyelination (25). The most frequent changes on RNFL in acute optical neuritis were observed in the temporal area (14). The stabilization of RNFL thickness occurs within 7-12 months from the beginning of the disease (23). Unless an adequate improvement of sight in patients with more significant RNFL thinning occurs within 3-6 months from the development of optical neuritis, subsequent improvement of sight can not be anticipated; therefore, all therapeutic procedures should be undertaken within the first six months after the outbreak of optical neuritis (24).

\section{RNFL thickness and visual functions after recovery from isolated optical neuritis}

In the examination of visual functions after recovery from isolated optical neuritis, Costello et al. suggested a correlation 


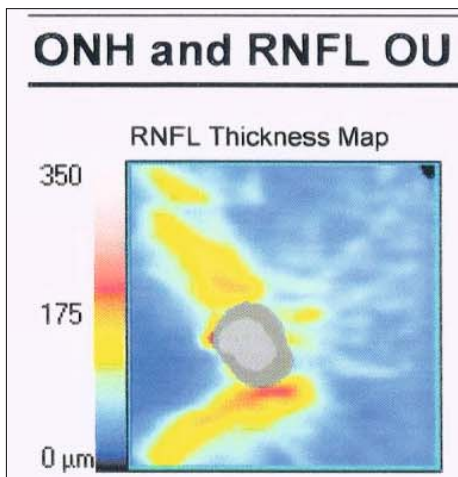

RNFL Deviation Map

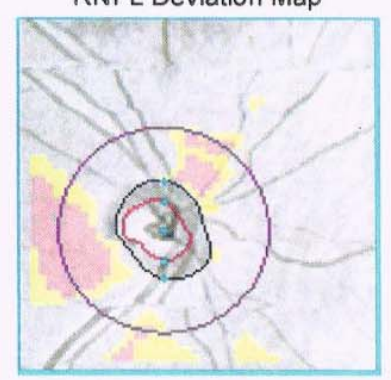

Disc Center $(-0.60,-0.54) \mathrm{mm}$

Extracted Horizontal Tomogram

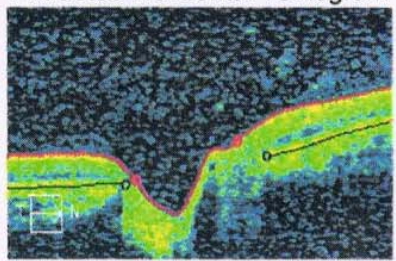

Extracted Vertical Tomogram

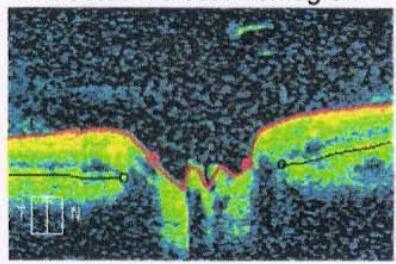

RNFL Circular Tomogram

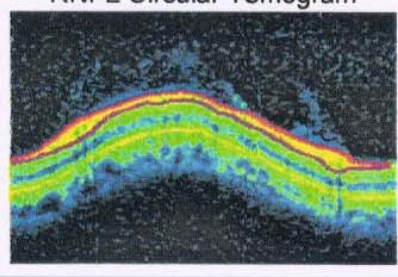

\begin{tabular}{|r|c|c|}
\hline & OD & OS \\
\hline Average RNFL Thickness & $78 \mu \mathrm{m}$ & $89 \mu \mathrm{m}$ \\
\hline RNFL Symmetry & \multicolumn{2}{|c|}{$88 \%$} \\
\hline Rim A.rea & $1.12 \mathrm{~mm}^{2}$ & $1.20 \mathrm{~mm}^{2}$ \\
\hline Disc Area & $1.95 \mathrm{~mm}^{2}$ & $1.76 \mathrm{~mm}^{2}$ \\
\hline Average CD Ratio & 0.64 & 0.55 \\
\hline Vertical CD Ratio & 0.60 & 0.52 \\
\hline Cup Volume & $0.207 \mathrm{~mm}^{3}$ & $0.118 \mathrm{~mm}^{3}$ \\
\hline
\end{tabular}

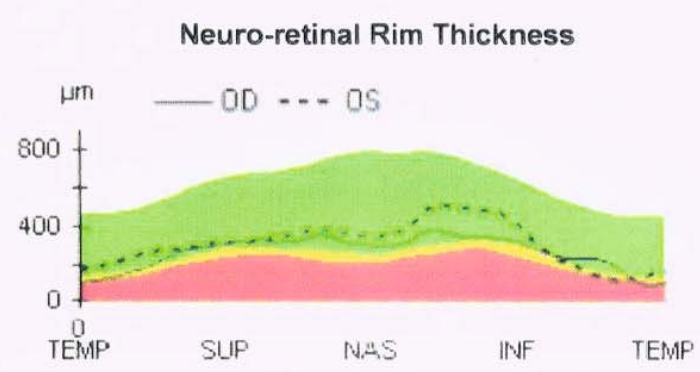

RNFL Thickness
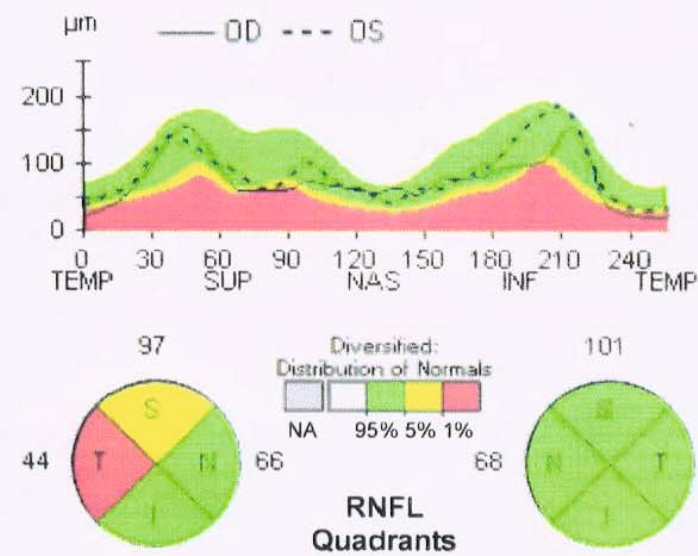

104

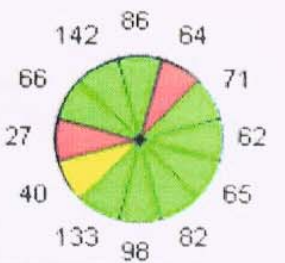

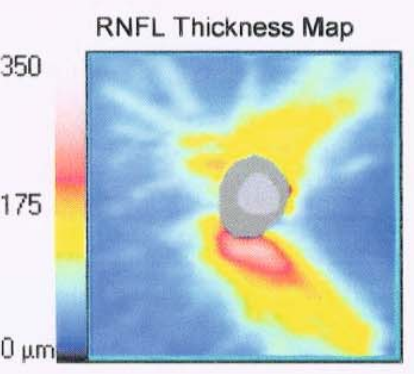

RNFL Deviation Map

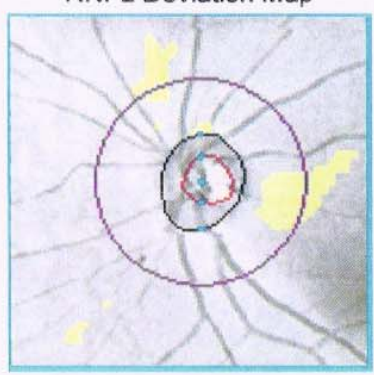

Disc Center $(0.15,0.21) \mathrm{mm}$ Extracted Horizontal Tomogram

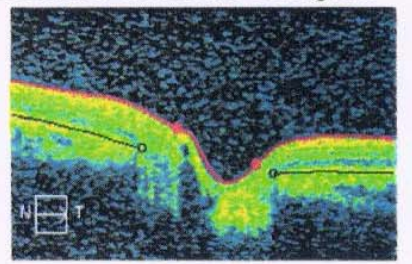

Extracted Vertical Tomogram

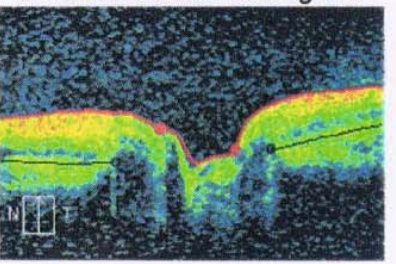

RNFL Circular Tomogram

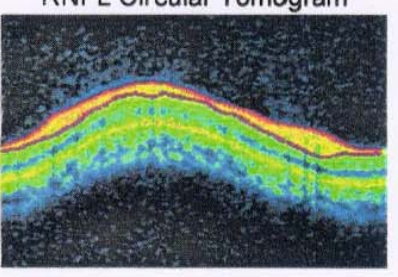

Fig. 1. An OCT RNFL report generated by Zeiss Cirrus spectral-domain OCT in a patient with MS.

between RNFL thickness and visual acuity and visual field. There was a linear correlation between RNFL thickness and visual acuity when RNFL measurements were under $70 \mu \mathrm{m}$; there was also a linear correlation between RNFL thickness and the average deviation in the Humphrey visual field, but only with RNFL measurements under $75 \mu \mathrm{m}$. The studies designated $75 \mu \mathrm{m}$ as threshold
RNFL thickness, with decrease in visual functions occurring with lower values $(23,25)$.

RNFL thickness in optical neuritis patients' asymptomatic eye

Numerous studies have shown that the thinning of RNFL was observed not only in the eyes with a previous episode of optical 
neuritis, but also in the seemingly asymptomatic opposite eyes of MS patients with a previous episode of optical neuritis, as well as in MS patients with no recorded clinical case of acute optical neuritis $(26,27,28,29,30,31)$. The average RNFL thickness was determined to be between 91.08 and $109.3 \mu \mathrm{m}$ in the opposite eye of patients with MS and optical neuritis $(20,21,22,26$, $30,32,33,34,35,36,37,38,39,40,41,42)$. In MS patients with no clinical record of optical neuritis, the average RNFL thickness was between 93.9 and $110.9 \mu \mathrm{m}(28,30,36,40,43)$. These findings are consistent with the previous records of lasting subclinical structural damage and axon loss, which was observed in magnetic resonance images of MS patients $(44,45)$. This supports the hypothesis that early treatment of MS can prevent progressive subclinical axon loss (46).

\section{OCT and multiple cclerosis}

\section{RNFL thickness and multiple sclerosis}

Very few studies were focused on quantifying RNFL thickness in patients with well-defined MS. In fact, most OCT studies included patients with and without a history of optical neuritis, and combined the results obtained on the opposite eye (considered as asymptomatic) of the patients with previous optical neuritis and the results from the eyes of patients with no history of optical neuritis. In spite of this, almost all of these studies proved that there was a thinning of RNFL in the eyes of MS patients when compared with the eyes of healthy subjects. The eyes with a previous episode of optical neuritis had a thinner RNFL than the unaffected eyes of MS patients, who also had a thinner RNFL than the eyes of healthy subjects (47).

\section{RNFL thickness after recovery from optical neuritis and the risk of multiple sclerosis}

In 2008, Costello et al (48) found that RNFL thickness after an episode of isolated optical neuritis can not be used to predict the risk of MS. During the average period of 34 months, they subsequently prospectively followed 50 patients with a single unilateral episode of optical neuritis with no history of MS, and compared RNFL thickness of affected and unaffected eyes during 1 or 2 years after the development of optical neuritis; in 21 out of the 50 patients, clinically definitive MS developed. After a year of following, the RNFL of the affected eyes was thicker in patients with clinically definitive MS $(89 \mu \mathrm{m})$ vs. the patients with no MS (78 $\mu \mathrm{m})$. The authors accounted for this surprising result by the fact that the study patients with no MS had more severe optical neuritis than the MS patients; this proves that RNFL thinning is mostly related to the severity of optical neuritis and the loss of sight and it does not help predict the subsequent risk of MS.

\section{RNFL thickness and the degree of severity of multiple sclerosis}

To a large extent, RNFL atrophy is connected with the physical damage evaluated on the Expanded Disability Status Scale (EDSS) $(19,31,49,50)$ and longer duration of the disease $(19,31)$. One of the studies, however, did not confirm any mutual relationship between RNFL thickness and EDSS (19). In a comparison of patients with an active course of the disease with neurologically stabilized patients, the RNFL in the temporal quadrant during the longer observation of MS patients thinned more significantly in the former group $(31,50)$. The average thinning of the total and temporal thickness of RNFL is lesser in patients who received immunomodulatory therapy within 0-24 months (31).

\section{RNFL thickness and the various forms of MS}

It appears that the rate of RNFL thinning observed in MS patients is affected by the subtype of MS. The patients with progressive forms of MS tend to suffer from a higher rate of RNFL thinning than patients with relapsing-remitting form of MS (RRMS) (30). Another study only found temporal quadrant RNFL thinning in patients with primary progressive MS (PPMS), while the group of patients with secondary progressive MS (SPMS) had a more significant RNFL thinning in the entire central, upper and temporal quadrants (36). When compared with healthy control subjects, the temporal quadrant was thinned in patients with PPMS, SPMS and also in clinically isolated syndromes (CIS). On the basis of these findings, it can be assumed that axon damage happens in the early stages of the disease (31). Costello et al (33) compared the RNFL thickness in patients with isolated optical neuritis, patients with RRMS and patients with SPMS, observed during a period of more than two years. The average RNFL thickness in eyes after an episode of optical neuritis was similar in all groups after one or two years of study, while temporal RNFL was thinner in both MS groups when compared with isolated optical neuritis patients. In unaffected eyes, the SPMS patients had a thinner RNFL after one and two years when compared with RRMS patients and isolated optical neuritis patients. After two years, the unaffected eyes of RRMS patients had thinner temporal RNFL than the unaffected eyes of isolated optical neuritis patients.

These results suggest that RNFL thickness measurements (mainly in the temporal quadrant) in the unaffected eyes of MS patients may help discern the MS subtype and predict the progression and severity of MS (51).

\section{RNFL thickness and visual evoked potentials}

The visual evoked potential (VEP) latency is more sensitive in expressing optic nerve damage than RNFL thickness (52). In studies, there was a strong correlation between RNFL thickness and VEP amplitude; however, the results of evaluation of the relationship between axon loss measured through OCT and VEP latency were contradictory $(20,53)$.

\section{RNFL thickness and magnetic resonance of the brain}

In MS patients, a large part of permanent invalidity is conditioned by axonal degeneration. Axonal loss happens as early as the first stages of MS (1,2). Even though conventional MRI techniques are sensitive to the inflammatory process (T2-weighted lesions), they do not specifically reflect axonal damage (54). MRI does not allow us to look into the process of subclinical optical neuropathy or to see the neurodegenerative effects on the retina, which are the result of optical neuropathy. RNFL thickness measurements using OCT could become a surrogate outcome marker for brain atrophy 
in MS (24). The process of RNFL thinning correlates with the atrophy of the optic nerve, which can be measured by conventional MRI devices (55) as well as by magnetization transfer ratio (MTR) $(31,56)$. In their study, Sepulcre et al $(31)$ showed a slightly significant correlation of RNFL thickness with the volume of white and gray brain matter, measured using conventional MRI, but not with the T1 and T2 volumes or the gadolinium enhanced lesions. A similar study proved that there was a relationship between RNFL thickness and the normalized volume of brain and white matter (40). The study also suggested that a larger number of T2 lesions correlated with thinner RNFL. The correlation between RNFL thickness and MRI measurements of brain atrophy was more significant in the subset of patients with no clinical history of optical neuritis than in those who suffered an episode of optical neuritis in the past (40). In yet another study, there was independent correlation between RNFL thickness and the brain parenchymal fraction (BPF) obtained using high-resolution anatomical MRI. This study also found that there was a correlation between BPF and EDSS (50). Later, a correlation between RNFL thickness, volume of T1 and T2 lesions, gray matter atrophy, MTR and diffusion tensor imaging measures (DTI) measurements in MS patients with or without a history of optical neuropathy was also discovered (34). These MRI parameters also correlated with low-contrast measurements of visual acuity, which are a sensitive marker of optic nerve dysfunction in MS. Gordon-Lipkin et al (54) showed that there was a relationship between RNFL thickness and overall brain atrophy. In MS patients, there was a significant relation between RNFL thickness, value of cerebral parenchyma component, volume of cerebrospinal fluid and EDSS score; it was not, however, related with the white and gray matter volumes. The authors believe that the results were partially influenced by the fact that the damage in the brain is first allocated to white matter. In subset analysis, a significant relationship was discovered in RRMS patients, but no such relationship was found in SPMS patients; this could be caused by the fact that most SPMS patients have more spinal lesions than cerebral ones.

\section{Can RNFL thickness be used as an outcome marker in clini- cal studies with MS?}

Some authors suggest that RNFL thickness measurements using OCT could be a better means to detect and monitor axon loss in MS than MRI. The interpretation of OCT exam results demands a good grasp of the technique and the device, as well as correlation with comprehensive eye examination. OCT resolution is more sensitive than brain MRI in evaluating early axon loss. As there is no myelin in the retina, RNFL measurements do not depend on myelin disorders and can reflect axon loss and thinning only. The fact that RNFL consists not only of axons but also of supporting tissue explains why the thickness of peripapillary RNFL in about $40 \mu \mathrm{m}$ even in patients with complete atrophy of the optic nerve atrophy and light sensitivity (57). It is comparatively easy to correlate OCT findings with visual functions measured using verified tools, like the visual acuity exam, low-contrast visual acuity measurements, contrast sensitivity, color perception, field of vision and visual evoked potential measurements. There is a relationship between RNFL thinning observed using OCT and abnormalities in visual functions $(23,25)$.

Ongoing studies and clinical trials now include measurements of RNFL thickness using standardized OCT protocols as their baseline measurements. If axon degeneration could be prevented using neuroprotectives, it could be expected to be reflected in unchanged RNFL thickness in OCT measurements. Even though there are estimates of potential RNFL thinning after episodes of acute optical neuritis in MS (23), it is equally important to know the estimated rate of RNFL thinning in MS without acute optical neuritis, in order to determine whether the neuroprotective drug in fact prevents axon degeneration. OCT has several important characteristics which make this imaging method a good potential surrogate outcome marker for the evaluation of the degree of axon damage in clinical studies.

\section{Conclusion}

OCT fulfills the requirement for a simple method which can evaluate axon integrity in MS through RNFL imaging. OCT can become a useful tool for following the progression of the disease in MS patients. It can improve our understanding of the effects of different therapies on MS patients and has the potential to become a surrogate outcome marker in studies examining the neuroprotective effect of drugs in MS. OCT can shed new light on the pathophysiology of MS, improve our understanding thereof, and thus contribute to the improvement in the clinical treatment of MS.

\section{References}

1. De Stefano N, Narayanan S, Francis GS et al. Evidence of axonal damage in the early stages of multiple sclerosis and its relevance to disability. Arch Neurol 2001; 58: 65-70.

2. Filippi M, Rocca MA. MRI aspects of the "inflammatory phase" of multiple sclerosis. Neurol Sci 2003; 24: 275-278.

3. Petzold A, de Boer JF, Schippling S et al. Optical coherence tomography in multiple sclerosis: a systematic review and meta-analysis. The Lancet Neurology, 2010; 9: 921-932.

4. Lamirel C, Newman NJ, Biousse V. The Use of Optical Coherence Tomography in Neurology. Rev Neurol Dis 2009; 6: 105-120.

5. Chen J, Lee L. Clinical applications and new developments of optical coherence tomography: an evidence-based review. Clin Exp Ophthalmol 2007; 90 (5): 317-335.

6. Sakata LM, DeLeon-Ortega J, Girkin CHA. Optical coherence tomography of the retina and optic nerve - a review. Clin Exp Ophthalmol 2009; 37: 90-99.

7. Swanson EA, Izatt JA, Hee MR, Huang D, Lin CP, Schuman JS et al. In vivo retinal imaging by optical coherence tomography. Opt Lett 1993; 18: 1864-1866.

8. Heckenlively JR, Arden GB (Eds). Anatomy of the retina, principles of cell biology in the visual pathways. Principles and practices of clinical electrophysiology of vision. Mosby Year Book Inc, 1991:21-37.

9. Rodieck RW (Ed). The vertebrate retina: principles of structure and function. WH Freeman and Company, San Francisco, CA. 1973: 338-343. 
10. Perry VH, Lund RD. Evidence that the lamina cribrosa prevents intraretinal myelination of retinal ganglion cell axons. J Neurocytol 1990; 19: $265-272$

11. Balcer LJ. Clinical practice. Optic neuritis. N Engl J Med 2006; 354 : $1273-1280$

12. Frohman EM, Frohman TC, Zee DS et al. The neuroophthalmology of multiple sclerosis. Lancet Neurol 2005; 4: 111-121.

13. Toussaint $D$, Périer $O$, Verstappen A et al. Clinicopathological study of the visual pathways, eyes, and cerebral hemispheres in 32 cases of disseminated sclerosis. J Clin Neuroophthalmol 1983; 3: 211-220.

14. Ikuta F, Zimmerman HM. Distribution of plaques in seventy autopsy cases of multiple sclerosis in the United States. Neurology 1976; 26: 26-28.

15. Moura FC, Medeiros FA, Monteiro ML. Evaluation of macular thickness measurements for detection of band atrophy of the optic nerve using optical coherence tomography. Ophthalmology 2007; 114: 175-181.

16. Burkholder BM, Osborne B, Loguidice MJ et al. Macular volume determined by optical coherence tomography as a measure of neuronal loss in multiple sclerosis. Arch Neurol 2009; 66: 1366-1372.

17. Kanamori A, Escano MF, Eno A et al. Evaluation of the effect of aging on retinal nerve fiber layer thickness measured by optical coherence tomography. Ophthalmologica 2003; 217: 273-278.

18. Brodsky M, Nazarian S, Orengo-Nania S et al. Multiple sclerosis risk after optic neuritis: final optic neuritis treatment trial follow-up. Arch Neurol 2008; 65 (6): 727-732.

19. Parisi V, Manni G, Spadaro M et al. Correlation between morphological and functional retinal impairment in multiple sclerosis patients. Invest Ophthalmol Vis Sci 1999; 40: 2520-2527.

20. Trip SA, Schlottmann PG, Jones SJ et al. Retinal nerve fiber layer axonal loss and visual dysfunction in optic neuritis. Ann Neurol 2005; 58: $383-391$.

21. Pro MJ, Pons ME, Liebmann JM, Ritch R, Zafar S, Lefton D et al. Imaging of the optic disc and retinal nerve fiber layer in acute optic neuritis. J Neurol Sci 2006; 250: 114-119.

22. Noval S, Contreras I, Rebolleda G, Munoz-Negrete FJ. Optical coherence tomography versus automated perimetry for follow-up of optic neuritis. Acta Ophthalmol Scand 2006; 84: 790-794.

23. Costello F, Hodge W, Pan YI, Eggenberger E, Coupland S, Kardon RH. Tracking retinal nerve fiber layer loss after optic neuritis: a prospective study using optical coherence tomography. Mult Scler 2008a; 14: 893-905.

24. Fisher JB, Jacobs DA, Markowitz CE, Galetta SL, Volpe NJ, Nano-Schiavi ML et al. Relation of visual function to retinal nerve fiber layer thickness in multiple sclerosis. Ophthalmology 2006; 113: 324-332.

25. Costello F, Coupland S, Hodge W, Lorello GR, Koroluk J, Pan YI et al. Quantifying axonal loss after optic neuritis with optical coherence tomography. Ann Neurol 2006; 59: 963-969.

26. Fisher JB, Jacobs DA, Markowitz CE, Galetta SL, Volpe NJ, Nano-Schiavi ML et al. Relation of visual function to retinal nerve fiber layer thickness in multiple sclerosis. Ophthalmology 2006; 113: 324-332.

27. Henderson AP, Trip SA, Schlottmann PG, Altmann DR, Garway-Heath DF, Plant GT et al. An investigation of the retinal nerve fibre layer in progressive multiple sclerosis using optical coherence tomography. Brain 2008; 131: 277-287.

28. Jeanjean L, Castelnovo G, Carlander B, Villain M, Mura F, Dupeyron $\mathrm{G}$ et al. Retinal atrophy using optical coherence tomography $(\mathrm{OCT})$ in
15 patients with multiple sclerosis and comparison with healthy subjects. Rev Neurol 2008; 164: 927-934.

29. Pueyo V, Martin J, Fernandez J, Almarcegui C, Ara J, Egea C et al. Axonal loss in the retinal nerve fiber layer in patients with multiple sclerosis. Mult Scler 2008; 14: 609-614.

30. Pulicken M, Gordon-Lipkin E, Balcer LJ, Frohman E, Cutter G, Calabresi PA. Optical coherence tomography and disease subtype in multiple sclerosis. Neurology 2007; 69: 2085-2092.

31. Sepulcre J, Murie-Fernandez M, Salinas-Alaman A, Garcia-Layana A, Bejarano B, Villoslada P. Diagnostic accuracy of retinal abnormalities in predicting disease activity in MS. Neurology 2007; 68: 1488-1494.

32. Cheng H, Laron M, Schiffman JS, Tang RA, Frishman LJ. The relationship between visual field and retinal nerve fiber layer measurements in patients with multiple sclerosis. Invest Ophthalmol Vis Sci 2007; 48: 5798-5805.

33. Costello F, Hodge W, Pan YI, Freedman M, DeMeulemeester C. Differences in retinal nerve fiber layer atrophy between multiple sclerosis subtypes. J Neurol Sci 2009; 281: 74-79.

34. Frohman EM, Dwyer MG, Frohman T, Cox JL, Salter A, Greenberg BM et al. Relationship of optic nerve and brain conventional and non-conventional MRI measures and retinal nerve fiber layer thickness, as assessed by OCT and GDx: a pilot study. J Neurol Sci 2009; 282: 96-105.

35. Grazioli E, Zivadinov R, Weinstock-Guttman B, Lincoff N, Baier M, Wong JR et al. Retinal nerve fiber layer thickness is associated with brain MRI outcomes in multiple sclerosis. J Neurol Sci 2008; 268: 12-17.

36. Henderson AP, Trip SA, Schlottmann PG, Altmann DR, GarwayHeath DF, Plant GT et al. An investigation of the retinal nerve fibre layer in progressive multiple sclerosis using optical coherence tomography. Brain 2008; 131: 277-287.

37. Klistorner A, Arvind H, Nguyen T, Garrick R, Paine M, Graham S et al. Multifocal VEP and OCT in optic neuritis: a topographical study of the structure-function relationship. Doc Ophthalmol 2009; 118: 129-137.

38. Ratchford JN, Quigg ME, Conger A, Frohman T, Frohman E, Balcer LJ et al. Optical coherence tomography helps differentiate neuromyelitis optica and MS optic neuropathies. Neurology 2009; 73: 302-308.

39. Rebolleda G, Munoz-Negrete FJ. Follow-up of Mild Papilledema in Idiopathic Intracranial Hypertension with Optical Coherence Tomography. Invest Ophthalmol Vis Sci 2008; 50: 5197-5200.

40. Siger M, Dziegielewski K, Jasek L, Bieniek M, Nicpan A, Nawrocki J et al. Optical coherence tomography in multiple sclerosis : Thickness of the retinal nerve fiber layer as a potential measure of axonal loss and brain atrophy. J Neurol 2008; 255: 1555-1560.

41. Spain RI, Maltenfort M, Sergott RC, Leist TP. Thickness of retinal nerve fiber layer correlates with disease duration in parallel with corticospinal tract dysfunction in untreated multiple sclerosis. J Rehabil Res Dev 2009; 46: 633-642.

42. Zaveri MS, Conger A, Salter A, Frohman TC, Galetta SL, Markowitz CE et al. Retinal imaging by laser polarimetry and optical coherence tomography evidence of axonal degeneration in multiple sclerosis. Arch Neurol 2008; 65: 924-928.

43. Gundogan FC, Demirkaya S, Sobaci G. Is optical coherence tomography really a new biomarker candidate in multiple sclerosis? A structural and functional evaluation. Invest Ophthalmol Vis Sci 2007; 48: 5773-5781.

44. De Stefano N, Matthews PM, Fu L, Narayanan S, Stanley J, Francis GS et al. Axonal damage correlates with disability in patients with 
relapsing-remitting multiple sclerosis. Results of a longitudinal magnetic resonance spectroscopy study. Brain 1998; 121: 1469-1477.

45. Narayanan S, Fu L, Pioro E, De Stefano N, Collins DL, Francis GS et al. Imaging of axonal damage in multiple sclerosis: spatial distribution of magnetic resonance imaging lesions. Ann Neurol 1997; 41: 385-391.

46. Thrower BW. Clinically isolated syndromes: predicting and delaying multiple sclerosis. Neurology 2007; 68: S12-15.

47. Burkholder BM, Osborne B, Loguidice MJ, Bisker E, Frohman TC, Conger A et al. Macular volume determined by optical coherence tomography as a measure of neuronal loss in multiple sclerosis. Arch Neurol 2009; 66: 1366-1372.

48. Costello F, Hodge W, Pan YI, Metz L, Kardon RH. Retinal nerve fiber layer and future risk of multiple sclerosis. Can J Neurol Sci 2008b; 35: $482-487$.

49. Gordon-Lipkin E, Chodkowski B, Reich DS, Smith SA, Pulicken $\mathbf{M}$ et al. Retinal nerve fiber layer is associated with brain atrophy in multiple sclerosis. Neurology 2007; 69: 1603-1609.

50. Toledo J, Sepulcre J, Salinas-Alaman A et al. Retinal nerve fiber layer atrophy is associated with physical and cognitive disability in multiple sclerosis Mult Scler 2008; 14: 906-912.

51. Lamirel C, Newman NJ, Biousse V. Optical Coherence Tomography (OCT) in Optic Neuritis and Multiple Sclerosis. Rev Neurol 2010; 166 (12): 978-986.
52. Naismith RT, Tutlam NT, Xu J et al. Optical coherence tomography is less sensitive than visual evoked potentials in optic neuritis. Neurology 2009; 73: 46-52.

53. Klistorner A, Arvind H, Nguyen T, Garrick R, Paine M et al. Axonal loss and myelin in early ON loss in postacute optic neuritis. Ann Neurol 2008; 64: 325-331.

54. Gordon-Lipkin E, Chodkowski B, Reich DS, Smith SA, Pulicken M et al. Retinal nerve fiber layer is associated with brain atrophy in multiple sclerosis. Neurology, 2007; 69: 1603-1609.

55. Trip SA, Schlottmann PG, Jones SJ, Li WY, Garway-Heath DF, Thompson AJ et al. Optic nerve atrophy and retinal nerve fibre layer thinning following optic neuritis: evidence that axonal loss is a substrate of MRI-detected atrophy. Neuroimage 2006; 31: 286-293.

56. Trip SA, Schlottmann PG, Jones SJ, Li WY, Garway-Heath DF, Thompson AJ et al. Optic nerve magnetization transfer imaging and measures of axonal loss and demyelination in optic neuritis. Mult Scler 2007; 13: 875-879.

57. Hood DC, Anderson SC, Wall M, Raza AS, Kardon RH. A Test of a Linear Model of Glaucomatous Structure-Function Loss Reveals Sources of Variability in Retinal Nerve Fiber and Visual Field Measurements. Invest Ophthalmol Vis Sci 2009; 50: 4254-4266.

58. Ratchford JN, Saidha S, Sotirchos ES et al. Active MS is associated with accelerated retinal ganglion cell/inner plexiform layer thinning. Neurology 2013; 80: 47-54. 